\title{
Identification and bioinformatics analysis of miRNAs associated with human muscle invasive bladder cancer
}

\author{
LIANG CHEN $^{1 *}$, LUSHUN YUAN $^{1 *}$, GANG WANG $^{1}$, RUI CAO $^{1}$, JIANPING PENG $^{1}$, \\ BO SHU $^{1,2}$, GUOFENG QIAN ${ }^{3}$, XINGHUAN WANG ${ }^{1}$ and YU XIAO ${ }^{1,4}$
}

\author{
${ }^{1}$ Department of Urology, Zhongnan Hospital of Wuhan University; ${ }^{2}$ Department of Urology, The Central Hospital of \\ Wuhan, Tongji Medical College, Huazhong University of Science and Technology, Wuhan, Hubei 430071; \\ ${ }^{3}$ Department of Endocrinology, The First Affiliated Hospital of Zhejiang University, Hangzhou, Zhejiang 310000; \\ ${ }^{4}$ Laboratory of Precision Medicine, Zhongnan Hospital of Wuhan University, Wuhan, Hubei 430071, P.R. China
}

Received February 14, 2017; Accepted August 11, 2017

DOI: $10.3892 / \mathrm{mmr} .2017 .7726$

\begin{abstract}
Accumulated evidence has indicated that micro (mi)RNAs play vital roles in the occurrence and development of human muscle invasive bladder cancer (MIBC), however, little is known about the miRNAs' regulatory networks. In the present study, the authors aimed to use bioinformatics analysis to identify the key miRNAs and potential target genes, as well as studying the underlying mechanisms for MIBC. They collected several human MIBC tissues to generate a miRNA expression analysis by microarray analysis comparing with normal bladder tissues, identifying 104 differentially expressed miRNAs (102 were downregulated and 2 were upregulated) and predicted 11,884 putative target genes of the dysregulated miRNAs. To understand the function of dysregulated miRNAs in the development of MIBC, networks among miRNAs and genes, gene ontologies and pathways were built. The subsequent bioinformatics analysis indicated that the mitogen-associated protein kinase (MAPK) signaling pathway, apoptosis and pathways in cancer and the cell cycle, were significantly enriched Overall, these results provided comprehensive information on the biological function of dysregulated miRNAs in the development of MIBC. The identification of miRNAs and their putative targets may offer new diagnostic and therapeutic strategies for human muscle invasive bladder cancer.
\end{abstract}

Correspondence to: Dr Xinghuan Wang or Dr Yu Xiao, Department of Urology, Zhongnan Hospital of Wuhan University, 169 Donghu Road, Wuhan, Hubei 430071, P.R. China

E-mail:wangxinghuan@whu.edu.cn

E-mail:yu.xiao@whu.edu.cn

*Contributed equally

Key words: bioinformatics analysis, muscle invasive bladder cancer, microRNA, microarray, miRNAs-gene-networks, miRNAs-GO-networks

\section{Introduction}

Human bladder cancer (BCa), one of the most common genitourinary malignancies arising from mucous membrane, accounts for $>3 \%$ of all malignant tumors around the world and more and more people are diagnosed each year (1). Early stage of bladder cancers do not usually cause symptoms and, with regard to those of late stage, it still frequently recurs and gradually progresses into muscle invasive $\mathrm{BCa}(2)$. The gold standard of $\mathrm{BCa}$ diagnosis is based on cystoscopy, which is invasive and relatively expensive. Currently, non-invasive and specific markers are used for urinary cytology, however, this method is not sensitive to detection of low-grade BCa. Thus, new, highly sensitive and specific urine-based diagnostic tools are particularly attractive because urine is a promising and readily available source for molecular markers, including RNA.

As small nucleotides of RNA, by binding to complementary sequences in the 3'-untranslated regions of specific mRNAs, micro (mi)RNAs inhibit the translation of specific target genes (3). In previous years, many cardinal cellular and physiological processes have been demonstrated to be associated with altered change in miRNAs expression levels (4-6). miRNAs are involved in the regulation of various cellular processes including cellular differentiation, cell cycle progression and apoptosis. Moreover, miRNAs, as important factors in tumorigenesis and metastasis and their expression signatures are associated with the prognosis and progression in a variety of cancers (7-9). Therefore, the authors could suppose that those miRNAs of which the expressions are changed significantly in tumors relative to normal tissues may have influence on tumor progression (10). Moreover, identifying target genes united with differentially expressed miRNAs might illustrate the roles of miRNAs in cancer biology (11). Previous studies have reported that we could distinguish malignant or normal tissue, as well as various tumor entities by miRNA expression profiles (12-16). As highly stable molecules, the authors could quantify miRNAs in tissues and body fluids, which makes them considered as promising cancer biomarkers. It has been reported that diagnosis, stage and sub-classification of cancer can be explored by differentially expressed miRNAs, which 
also can predict treatment efficacy and prognosis (4). However, the molecular mechanisms are not yet clearly elucidated, which makes it necessary to identify novel miRNAs.

In the present study, the group performed an MIBC-related miRNA expression profile, so as to address the functional roles of miRNAs in MIBC. Several human MIBC tissues and normal bladder tissues were conducted to conduct a microarray analysis (GEO accession No. GSE76211), revealing several differentially expressed miRNAs. The putative target genes of dysregulated miRNAs were predicted using miRNA databases. Then the networks among miRNAs and genes, gene ontologies (GOs) and pathways were built. The purpose of the current study was to identify candidate predictive tumor-associated miRNAs in MIBC patients.

\section{Materials and methods}

Preparation for human bladder samples. Three MIBC tissues samples were collected from patients after surgery at Zhongnan Hospital of Wuhan University (Wuhan, China). Three normal bladder tissue samples were collected from donors by accidental death. Information of the MIBC patients and donors was listed in Table I. Those samples used in the study have been described in previous publication (17-19). Briefly, two pathologists confirmed the histology diagnosis independently and all the tissues were snap-frozen for total RNA isolation at liquid nitrogen after excision from operation room. Informed consent was obtained for surgery patients and normal donors from the patients and their relatives, respectively. The Ethics Committee at Zhongnan Hospital of Wuhan University (Wuhan, China) approved the experiments using human bladder tissue samples for RNA isolation analysis (approval number: 2015029). All methods used for human bladder tissue samples were performed in accordance with the approved guidelines and regulations.

RNA extraction. Based on the manufacturer's protocol, total RNA was extracted from the frozen tissue block using RNeasy Mini kit (cat. no. 74101, Qiagen GmbH, Hilden, Germany), combined with QIAshredder (cat. no. 79654, Qiagen $\mathrm{GmbH}$ ) using a centrifuge (cat. no. 5424, Eppendorf, Hamburg, Germany). In order to remove genomic DNA, DNase I digestion (cat. no. 79253, Qiagen GmbH), DNase I digestion (cat. no. 79254, Qiagen $\mathrm{GmbH}$ ) was used in each RNA preparation.

miRNA microarray. After assessing RNA quality and quantity, the miRNAs microarray analysis (Affymetrix microRNA 4.0 Array, Affymetrix, Inc., Santa Clara, CA, USA) was performed according to the manufacturer's instructions. Briefly, $1 \mu \mathrm{g}$ of total RNA was labeled with Biotin using the FlashTag Biotin HSR RNA Labeling kit (Genisphere LLC, Hatfield, PA, USA) and then hybridized overnight with the array, which was washed, stained, and read by an GeneChip Scanner 3000 7G (Affymetrix, Inc.). MiRNA microarray data GSE40355 used for validation were obtained from Gene Expression Omnibus (GEO) database (http://www.ncbi.nlm.nih.gov/geo/). This dataset included eight normal bladder tissues samples, eight low grade $\mathrm{BCa}$ tissues samples and eight high grade $\mathrm{BCa}$ tissue samples. Among them, significant expressed miRNAs were screened out from high grade BCa tissues compared with normal bladder tissues.

Data analysis of miRNAs microarray. CEL-files of the raw data were first exported by Affymetrix GeneChip Command Console Software Version 4.0 (Affymetrix, Inc.) and then uploaded to the website of Gminix-Cloud Biotechnology Information (GCBI) by Genminix Informatics Co., Ltd. (Shanghai, China; http://www.gcbi. com.cn/gclib/html/index) for further analysis, including difference analysis of miRNAs profiles, prediction of miRNAs target genes, GO/pathway enrichment analysis, miRNAs-gene-network and miRNAs-GO-network analysis. The miRNAs array data used in the present paper has been uploaded to the NCBI Gene Expression Omnibus and the GEO accession number is GSE76211.

According to the GCBI online method description for difference analysis, the procedure for candidate miRNAs selection is as follows: When the number of samples in each group is no less than 3, SAM method is used for difference analysis. The authors implemented a series of steps to obtain the estimation of significance of difference and false discovery rate for every filtered gene:

i) Calculate the exchange factor $\mathrm{s}_{0}$ : Firstly, calculate the standard deviation for all genes, denote $s^{\alpha}$ as the $\alpha$ percentile for $\mathrm{s}_{\mathrm{i}}$. For the percentile value $\mathrm{q}_{1}<\mathrm{q}_{2} \ldots \mathrm{q}_{100}$ of the $\mathrm{s}_{\mathrm{i}}$, calculate the statistic:

$$
v_{j}=\operatorname{mad}\left\{d_{i}^{\alpha}=r_{i} /\left(s_{i}+s^{\alpha}\right) \mid s_{i}=\left[q_{j}, q_{j+1}\right)\right\}
$$

Where mad denotes the mean absolute deviation. At last, $\alpha$ (denote as $\hat{\alpha}$ ) was selected to make the CV (coefficient of variation) of the $\mathrm{v}_{\mathrm{j}}$ achieve minimum. Then, the exchange factor $s_{0}$ is $s^{\hat{\alpha}}$ used.

ii) Calculate the statistic value (d Score) for every gene:

$$
\mathrm{d}_{\mathrm{i}}=\mathrm{r}_{\mathrm{i}} /\left(\mathrm{s}_{\mathrm{i}}+\mathrm{s}_{0}\right)
$$

Where $r_{i}$ reflects the difference in average level among different groups, $\mathrm{s}_{\mathrm{i}}$ reflects the variation of sample population. See details in references $(20,21)$

iii) Calculate the order statistic:

$$
\mathrm{d}_{(1)} \leq \mathrm{d}_{(2)} \leq \cdots \leq \mathrm{d}_{(\mathrm{i})} \leq \cdots \leq \mathrm{d}_{(\mathrm{p})}
$$

iv) In order to get the above statistic's estimate, the authors made a permutation method (a loop strategy through every sample, the total number no less than 1,000; the detail are omitted) the expected distribution of $d$ score. The estimated statistic values are denoted as follows:

$$
\mathrm{d}_{(1)}^{*} \leq \mathrm{d}_{(2)}^{*} \leq \cdots \leq \mathrm{d}_{(\mathrm{i})}^{*} \leq \cdots \leq \mathrm{d}_{(\mathrm{p})}^{*}
$$

v) The authors obtain the order statistic value under the permutation:

$$
\overline{\mathrm{d}}_{(\mathrm{i})}=\frac{\sum_{\mathrm{i}=1}^{1000} \mathrm{~d}_{(\mathrm{i})}^{*}}{1000}
$$

vi) By calculating the maximum distance between the order statistic $\mathrm{d}_{(\mathrm{i})}$ and the expected order statistic $\overline{\mathrm{d}}_{(\mathrm{i})}$, the authors 
constructed a series of rejection regions for q-value. In fact, a grid of delta values was obtained by dividing 50 equivalent delta value for the above distance.

vii) For a fixed delta value, by computing the difference $\Delta_{(\mathrm{i})}=\mathrm{d}_{(\mathrm{i})}-\overline{\mathrm{d}}_{(\mathrm{i})}$ the authors identified the nearest $\Delta_{(\mathrm{i})}$ for gene $\mathrm{i}$. The cut-up is marked as min $\left\{\Delta_{(i)} \geq\right.$ delta $\}$ for positive gene and the cut-down $\max \left\{\Delta_{(\mathrm{i})} \leq\right.$ delta $\}$ as for negative gene. The genes with differences above the cut-up value (we denote the number of these genes as $\mathrm{R}_{(\mathrm{p})}$ ) were considered as significantly positive genes. While the genes with differences lower than the cut-down value were considered as significantly negative genes.

viii) Under the above cut-up and cut-down thresholds, the simulation of step VII was performed respectively on the statistics obtained from step V, such that the number of positive genes could be obtained under random state ( $\geq 1,000$ permutations). The median of the 1,000 positive genes was estimated as the number of false positive genes, to allow the false discovery rate (FDR) to be estimated (the positive $^{\mathrm{FDR}=\frac{V_{(p)}}{n_{(p)}}}$ and the negative FDR was similar), and thus the proportion of false positive genes in the full set of positive genes.

ix) Finally, according to the definition of q-value (22), the authors obtained the q-value for the gene, i, by selecting the minimum of the FDR for the 50 delta values determined in step VII (every delta as a rejection region).

\section{Results}

Identification of differentially expressed miRNAs (DE-miRNAs) in BCa tissues. The obtained miRNA expression profiles (GSE76211) of BCa and normal bladder tissues were analyzed by the Affymetrix microRNA 4.0 Array, which contains 2,578 probes and can interrogate all mature miRNAs sequences in miRBase Release 20. The results revealed that 104 miRNAs were dysregulated in BCa group under the condition of ' $\mathrm{P}<0.05$ and fold change $>1.5$ ', compared with normal bladder group. Among them, 102 miRNAs were downregulated and 2 were upregulated (Fig. 1). All of the dysregulated miRNA were listed in Table II.

Identification of putative target genes. The current study has identified 104 miRNAs that were significantly dysregulated in BCa tissues compared with normal bladder tissues. As miRNAs play their functional roles by regulating target genes expression at the posttranscriptional level, the authors predicted the target genes of dysregulated miRNAs using GCBI online tools, which were mainly based on the algorithms of miRanda and TargetScan. A total of 11,884 genes were predicted as putative target genes of dysregulated miRNAs.

GO/pathway enrichment analysis of putative target genes of dysregulated miRNAs. To understand the role of miRNAs in cancer development, GO and pathway enrichment analysis were performed. The data in Fig. 2A indicated that the top 10 dysregulated GOs were 'transcription, DNA-dependent', 'signal transduction, positive regulation of transcription from
Table I. Information of the patients and donors.

\begin{tabular}{lcc}
\hline Characteristics & MIBC patients & Donors \\
\hline Number & 3 & 3 \\
Age, years (mean \pm SD) & $62 \pm 1.581$ & $37 \pm 2.327$ \\
Gender & Male & Male \\
BCa stage & Stage II & - \\
Surgical method & Radical resection & - \\
\hline
\end{tabular}

Stage II denotes that BCa enters the muscle layer of the bladder. SD, standard deviation; MIBC, muscle invasive bladder cancer; $\mathrm{BCa}$, bladder cancer.

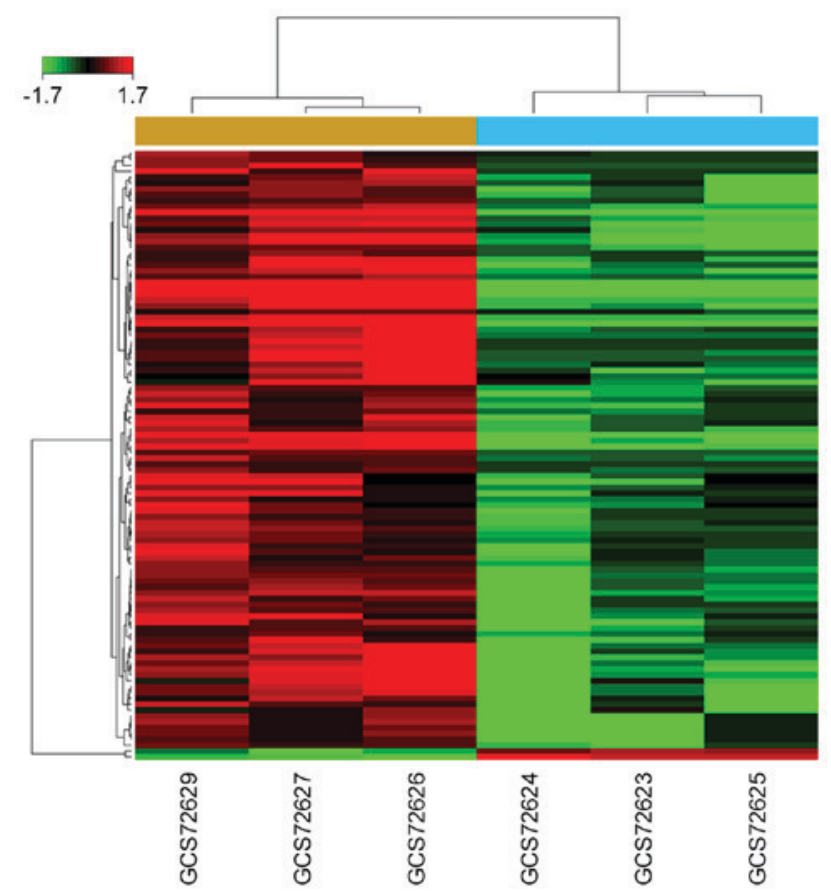

Figure 1. Differential expression of miRNAs between normal bladder and MIBC tissues. Heatmap of 104 microRNAs differentially expressed between three normal bladder and three MIBC tissues. MIBC, muscle invasive bladder cancer.

RNA polymerase II promoter', 'regulation of transcription, DNA-dependent', 'axon guidance', 'positive regulation of transcription, DNA-dependent', 'apoptotic process', 'negative regulation of transcription from RNA polymerase II promoter', 'synaptic transmission' and 'negative regulation of transcription, DNA-dependent'. GO analysis obviously suggests that many dysregulated miRNAs may contribute to tumorigenesis of bladder through many important functions such as transcription regulation, signal transduction as well as apoptotic process. Combined with the KEGG database, the authors analyzed the pathways involving the putative target genes. As illustrated in Fig. 2B, the top ten dysregulated pathways were the mitogen-associated protein kinase (MAPK) signaling pathway, apoptosis, pathways in cancer, cell cycle, p53 signaling pathway, calcium signaling pathway, Wnt signaling pathway, adherens junction, focal adhesion and ErbB signaling pathway. 
Table II. Differently expressed miRNAs in human MIBC tissues.

MIBC tissues vs. normal bladder tissues. $(\mathrm{P}<0.05$, Fold-change $>1.5)$

\begin{tabular}{|c|c|c|c|c|}
\hline miRNA & Change & P-value & Feature $^{a}$ & Rank $^{\mathrm{b}}$ \\
\hline hsa-miR-4786-5p & -10.205618 & 0.00124 & Down & 1 \\
\hline hsa-miR-490-3p & -35.773813 & 0.001358 & Down & 2 \\
\hline hsa-miR-3617-5p & -42.647285 & 0.001476 & Down & 3 \\
\hline hsa-miR-490-5p & -75.331847 & 0.001594 & Down & 4 \\
\hline hsa-miR-139-3p & -32.552806 & 0.001712 & Down & 5 \\
\hline hsa-miR-133b & -121.234878 & 0.00183 & Down & 6 \\
\hline hsa-miR-145-3p & -9.840205 & 0.001948 & Down & 7 \\
\hline hsa-miR-29b-1-5p & -10.581222 & 0.002066 & Down & 8 \\
\hline hsa-miR-155-5p & 8.720112 & 0.002184 & UP & 9 \\
\hline hsa-miR-1 & -40.712728 & 0.002302 & Down & 10 \\
\hline hsa-miR-548q & -5.779384 & 0.00242 & Down & 11 \\
\hline hsa-miR-133a-5p & -17.075477 & 0.002774 & Down & 12 \\
\hline hsa-miR-146b-5p & 4.140864 & 0.002893 & UP & 13 \\
\hline hsa-miR-28-3p & -3.834537 & 0.003129 & Down & 14 \\
\hline hsa-miR-30c-2-3p & -11.293849 & 0.003247 & Down & 15 \\
\hline hsa-miR-143-5p & -15.820855 & 0.003365 & Down & 16 \\
\hline hsa-miR-6511b-3p & -6.432579 & 0.003601 & Down & 17 \\
\hline hsa-miR-320e & -3.630028 & 0.003719 & Down & 18 \\
\hline hsa-miR-99a-3p & -2.325859 & 0.003837 & Down & 19 \\
\hline hsa-miR-30a-3p & -18.900354 & 0.003955 & Down & 20 \\
\hline hsa-miR-133a-3p & -63.82892 & 0.004073 & Down & 21 \\
\hline hsa-miR-4510 & -2.805811 & 0.004545 & Down & 22 \\
\hline hsa-miR-139-5p & -12.808018 & 0.004782 & Down & 23 \\
\hline hsa-miR-4324 & -19.035251 & 0.005136 & Down & 24 \\
\hline hsa-miR-145-5p & -3.499059 & 0.005372 & Down & 25 \\
\hline hsa-miR-125b-1-3p & -13.117689 & 0.005608 & Down & 26 \\
\hline hsa-miR-143-3p & -3.894089 & 0.005962 & Down & 27 \\
\hline hsa-miR-193a-5p & -4.050292 & 0.006198 & Down & 28 \\
\hline hsa-miR-5684 & -4.681531 & 0.007261 & Down & 29 \\
\hline hsa-miR-328-3p & -8.31845 & 0.007379 & Down & 30 \\
\hline hsa-miR-4429 & -2.76178 & 0.007497 & Down & 31 \\
\hline hsa-miR-1287-5p & -7.357196 & 0.007615 & Down & 32 \\
\hline hsa-miR-3605-5p & -2.739437 & 0.007969 & Down & 33 \\
\hline hsa-miR-338-5p & -4.852428 & 0.008323 & Down & 34 \\
\hline hsa-miR-193a-3p & -9.317892 & 0.00856 & Down & 35 \\
\hline hsa-miR-4257 & -3.696435 & 0.008796 & Down & 36 \\
\hline hsa-miR-6507-5p & -6.813232 & 0.010331 & Down & 37 \\
\hline hsa-miR-6722-3p & -2.904131 & 0.011275 & Down & 38 \\
\hline hsa-miR-497-5p & -3.273151 & 0.011865 & Down & 39 \\
\hline hsa-miR-125b-2-3p & -7.613095 & 0.012574 & Down & 40 \\
\hline hsa-miR-29b-2-5p & -2.995467 & 0.013046 & Down & 41 \\
\hline hsa-miR-6511a-3p & -8.396761 & 0.013282 & Down & 42 \\
\hline hsa-miR-628-3p & -4.613832 & 0.013636 & Down & 43 \\
\hline hsa-miR-378b & -6.024561 & 0.014581 & Down & 44 \\
\hline hsa-miR-664a-5p & -6.119249 & 0.014699 & Down & 45 \\
\hline hsa-miR-204-5p & -6.006885 & 0.015525 & Down & 46 \\
\hline hsa-miR-7641 & -4.116459 & 0.015762 & Down & 47 \\
\hline hsa-miR-320d & -2.149376 & 0.016706 & Down & 48 \\
\hline hsa-miR-3656 & -2.093619 & 0.017414 & Down & 49 \\
\hline hsa-miR-1273g-3p & -2.156204 & 0.017769 & Down & 50 \\
\hline
\end{tabular}


Table II. Continued.

\begin{tabular}{|c|c|c|c|c|}
\hline miRNA & Change & P-value & Feature $^{a}$ & $\operatorname{Rank}^{\mathrm{b}}$ \\
\hline hsa-miR-378g & -7.277976 & 0.018359 & Down & 51 \\
\hline hsa-miR-1225-5p & -2.552195 & 0.019067 & Down & 52 \\
\hline hsa-miR-3156-5p & -6.520017 & 0.019421 & Down & 53 \\
\hline hsa-miR-383-5p & -3.70084 & 0.019658 & Down & 54 \\
\hline hsa-miR-3064-3p & -2.07381 & 0.020012 & Down & 55 \\
\hline hsa-miR-378d & -5.5391 & 0.02013 & Down & 56 \\
\hline hsa-miR-23b-5p & -9.113128 & 0.020366 & Down & 57 \\
\hline hsa-miR-3195 & -10.384316 & 0.020484 & Down & 58 \\
\hline hsa-miR-100-5p & -3.57042 & 0.020956 & Down & 59 \\
\hline hsa-miR-378e & -14.003579 & 0.021547 & Down & 60 \\
\hline hsa-miR-4649-5p & -2.337674 & 0.022019 & Down & 61 \\
\hline hsa-miR-3622b-5p & -3.04368 & 0.022373 & Down & 62 \\
\hline hsa-miR-6840-3p & -6.817266 & 0.024616 & Down & 63 \\
\hline hsa-miR-4322 & -6.233632 & 0.024852 & Down & 64 \\
\hline hsa-miR-574-5p & -5.490031 & 0.025207 & Down & 65 \\
\hline hsa-miR-4770 & -4.247774 & 0.025561 & Down & 66 \\
\hline hsa-miR-513a-5p & -3.907964 & 0.025679 & Down & 67 \\
\hline hsa-miR-124-3p & -1.707013 & 0.025797 & Down & 68 \\
\hline hsa-miR-6819-5p & -2.385821 & 0.026151 & Down & 69 \\
\hline hsa-miR-339-3p & -5.134485 & 0.027332 & Down & 70 \\
\hline hsa-miR-26b-3p & -3.129563 & 0.02745 & Down & 71 \\
\hline hsa-miR-29c-5p & -7.931159 & 0.027922 & Down & 72 \\
\hline hsa-miR-29a-3p & -2.906434 & 0.02804 & Down & 73 \\
\hline hsa-miR-1227-5p & -2.287321 & 0.028276 & Down & 74 \\
\hline hsa-miR-4327 & -2.710984 & 0.028512 & Down & 75 \\
\hline hsa-miR-99a-5p & -4.947917 & 0.02863 & Down & 76 \\
\hline hsa-miR-1296-5p & -9.401257 & 0.029103 & Down & 77 \\
\hline hsa-miR-378i & -4.649838 & 0.030283 & Down & 78 \\
\hline hsa-miR-6889-5p & -4.810854 & 0.030401 & Down & 79 \\
\hline hsa-miR-186-5p & -3.276383 & 0.031464 & Down & 80 \\
\hline hsa-miR-6824-5p & -8.197661 & 0.031936 & Down & 81 \\
\hline hsa-miR-195-3p & -4.796311 & 0.034416 & Down & 82 \\
\hline hsa-miR-3622a-5p & -4.145204 & 0.034888 & Down & 83 \\
\hline hsa-miR-5572 & -6.053895 & 0.03536 & Down & 84 \\
\hline hsa-miR-6776-5p & -3.050977 & 0.035832 & Down & 85 \\
\hline hsa-miR-6790-5p & -2.868339 & 0.036895 & Down & 86 \\
\hline hsa-miR-6740-5p & -2.087867 & 0.037721 & Down & 87 \\
\hline hsa-miR-4269 & -8.226931 & 0.038312 & Down & 88 \\
\hline hsa-miR-125b-5p & -2.694152 & 0.039138 & Down & 89 \\
\hline hsa-miR-3188 & -8.008159 & 0.039256 & Down & 90 \\
\hline hsa-miR-6787-5p & -2.714687 & 0.03961 & Down & 91 \\
\hline hsa-miR-99b-5p & -1.811603 & 0.039847 & Down & 92 \\
\hline hsa-miR-30a-5p & -3.787576 & 0.040673 & Down & 93 \\
\hline hsa-miR-6765-5p & -2.365181 & 0.041027 & Down & 94 \\
\hline hsa-miR-1909-3p & -7.648533 & 0.041972 & Down & 95 \\
\hline hsa-miR-4507 & -2.786848 & 0.042326 & Down & 96 \\
\hline hsa-miR-28-5p & -2.529154 & 0.042916 & Down & 97 \\
\hline hsa-miR-6892-5p & -2.081233 & 0.046458 & Down & 98 \\
\hline hsa-miR-4433b-3p & -1.923165 & 0.046694 & Down & 99 \\
\hline hsa-miR-6737-5p & -3.166103 & 0.04693 & Down & 100 \\
\hline hsa-miR-324-3p & -3.939764 & 0.048465 & Down & 101 \\
\hline hsa-miR-211-3p & -3.140159 & 0.048819 & Down & 102 \\
\hline
\end{tabular}


Table II. Continued.

\begin{tabular}{lllr}
\hline miRNA & Change & P-value & Feature $^{\mathrm{a}}$ \\
\hline hsa-miR-6798-5p & -2.080154 & Down & 103 \\
hsa-miR-4299 & -4.93377 & 0.049174 & Down \\
\hline
\end{tabular}

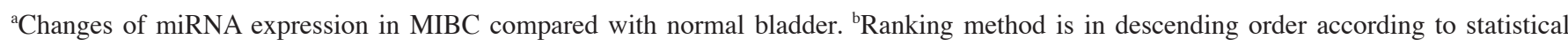
significance (P-value) between the two groups. miRNA/miR, microRNA; MIBC, muscle invasive bladder cancer; Up, upregulated in MIBC; Down, downregulated in MIBC.

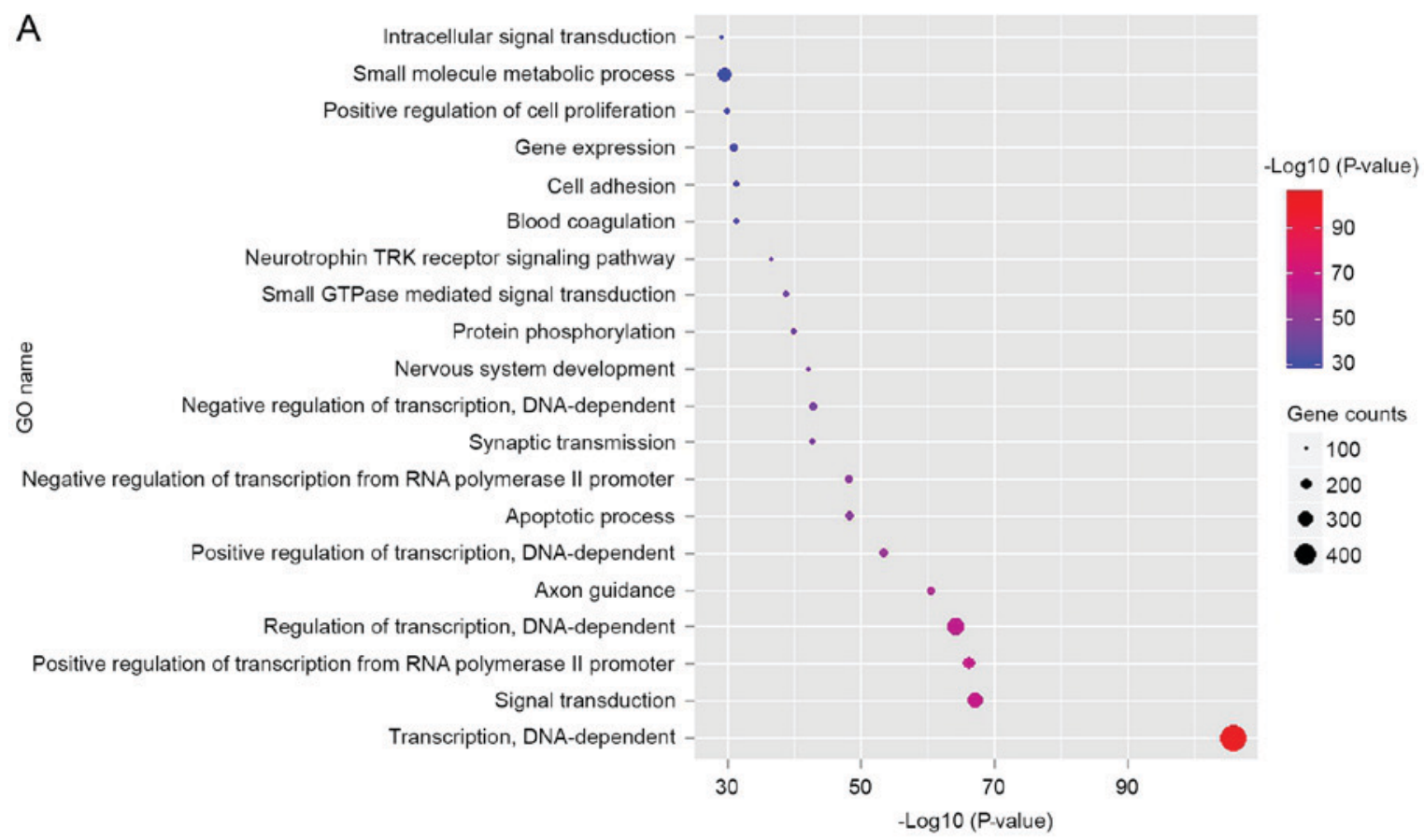

B

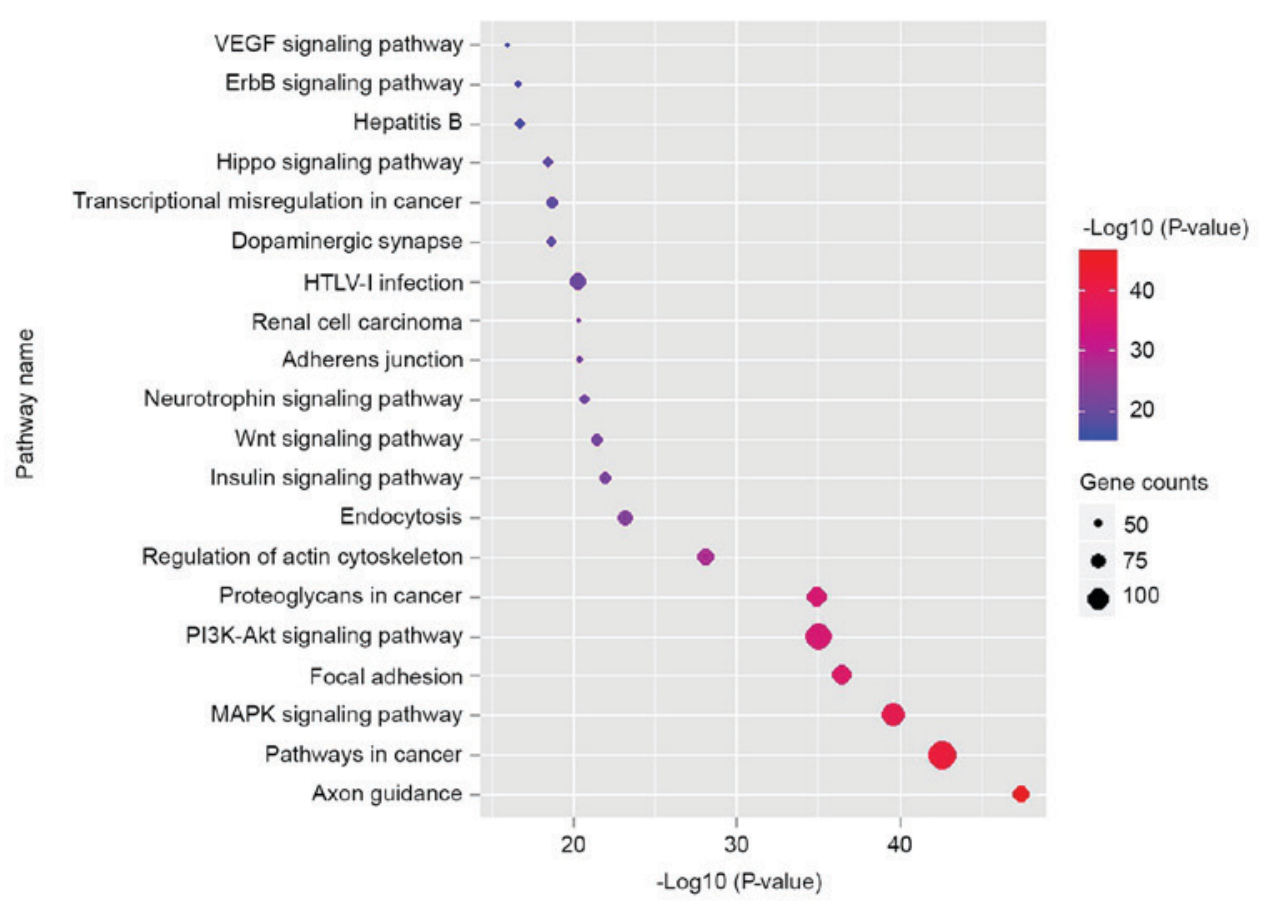

Figure 2. Significantly changed GO/pathways of predicted target genes of dysregulated miRNAs. (A) Significantly changed GOs of predicted target genes. The $y$-axis shows GO category and the $x$-axis shows $-\log 10$ (P-value). (B) Significantly changed pathways of predicted target genes. The $\gamma$-axis shows significantly changed pathways. GO, gene ontology. 


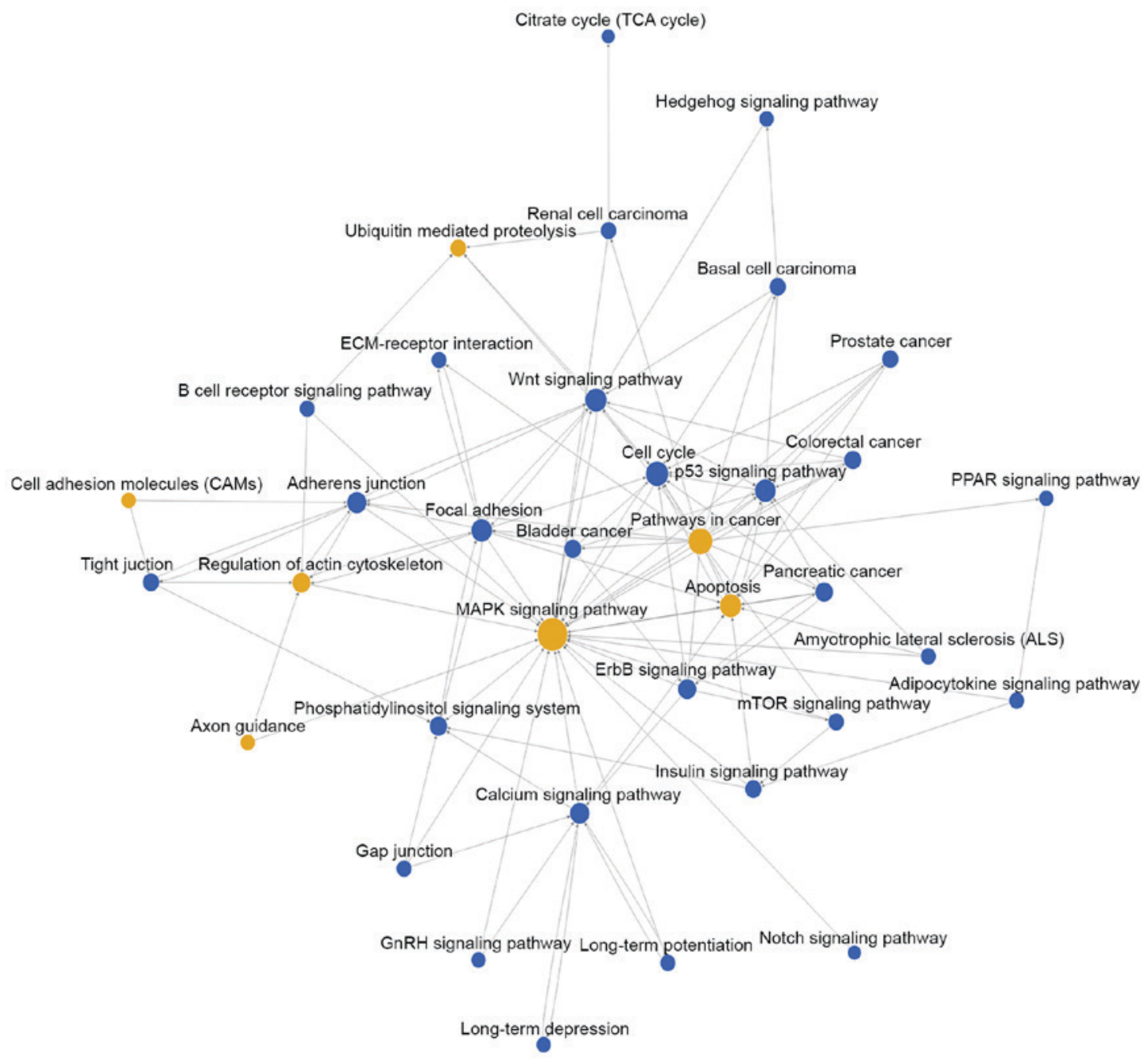

Figure 3. Pathway network (Path-net). Significantly changed pathways were connected in a Path-net to show the interaction network among these pathways. Each pathway in the network was measured by counting the upstream and downstream pathways. The blue circle represents pathways involving upregulated miRNAs, while the yellow circle represents pathways involving both upregulated and downregulated miRNAs. The size of the circle represents the degree value and the lines show the interaction between pathways. A higher degree of pathway indicates that it plays a more important role in the signaling network. miRNAs, microRNAs.

Pathway network analysis. Then, pathway network (Path-net) analysis was performed to draw an interaction network covering 36 significantly changed pathways (Fig. 3). Among them, the MAPK signaling pathway (degree $=44)$, apoptosis (degree $=30$ ), pathways in cancer $($ degree $=29)$ and cell cycle (degree $=24$ ) showed the highest degree, suggesting that these four pathways might play a core role in regulation of bladder cancer development.

miRNAs-gene-networks and miRNAs-GO-networks. Based on the significantly regulated GOs and pathways, the authors selected intersected genes and further constructed miRNAs-gene-networks and miRNAs-GO-networks to screen the key regulatory functions of the identified miRNAs and their target genes, respectively. As shown in Figs. 4 and 5, and Table III, the top rated nine miRNAs were hsa-miR-497-5p, hsa-miR-29a-3p, hsa-miR-124-3p, hsa-miR-4269, hsa-miR-145-5p, hsa-miR-204-5p, hsa-miR-4510, hsa-miR-6840-3p and hsa-miR-6722-3p. All of these miRNAs were downregulated in $\mathrm{BCa}$ tissues compared with normal bladder tissues. The microarray analysis demonstrated that the dysregulated miRNAs primarily play vital roles in various biological processes, including transcription regulation, apoptotic process, gene expression and signal transduction. Taken together, deregulation of certain miRNAs and several important pathways may be closely associated with human bladder cancer development.

Validation of candidate miRNAs. To confirm that the top rated nine miRNAs identified were indeed dysregulated in $\mathrm{BCa}$ tissues, the authors used another miRNA expression profiling GSE40355 including 8 normal bladder tissues samples, 8 low grade $\mathrm{BCa}$ tissues samples and 8 high grade $\mathrm{BCa}$ tissue samples for validation. In the dataset of GSE40355, the expression values of candidate miRNAs between normal bladder tissue samples with high grade $\mathrm{BCa}$ tissue samples were extracted for $\mathrm{t}$ test, and $\mathrm{P}<0.05$ were considered statistically significant. In addition, 157 dysregulated miRNAs including 69 upregulated and 88 downregulated miRNAs were obtained by analysis of GSE40355. As presented in Fig. 6 and Table IV, among the top rated nine miRNAs screened from our miRNA microarray, four miRNAs involving hsa-miR-497-5p, hsa-miR-29a-3p, 


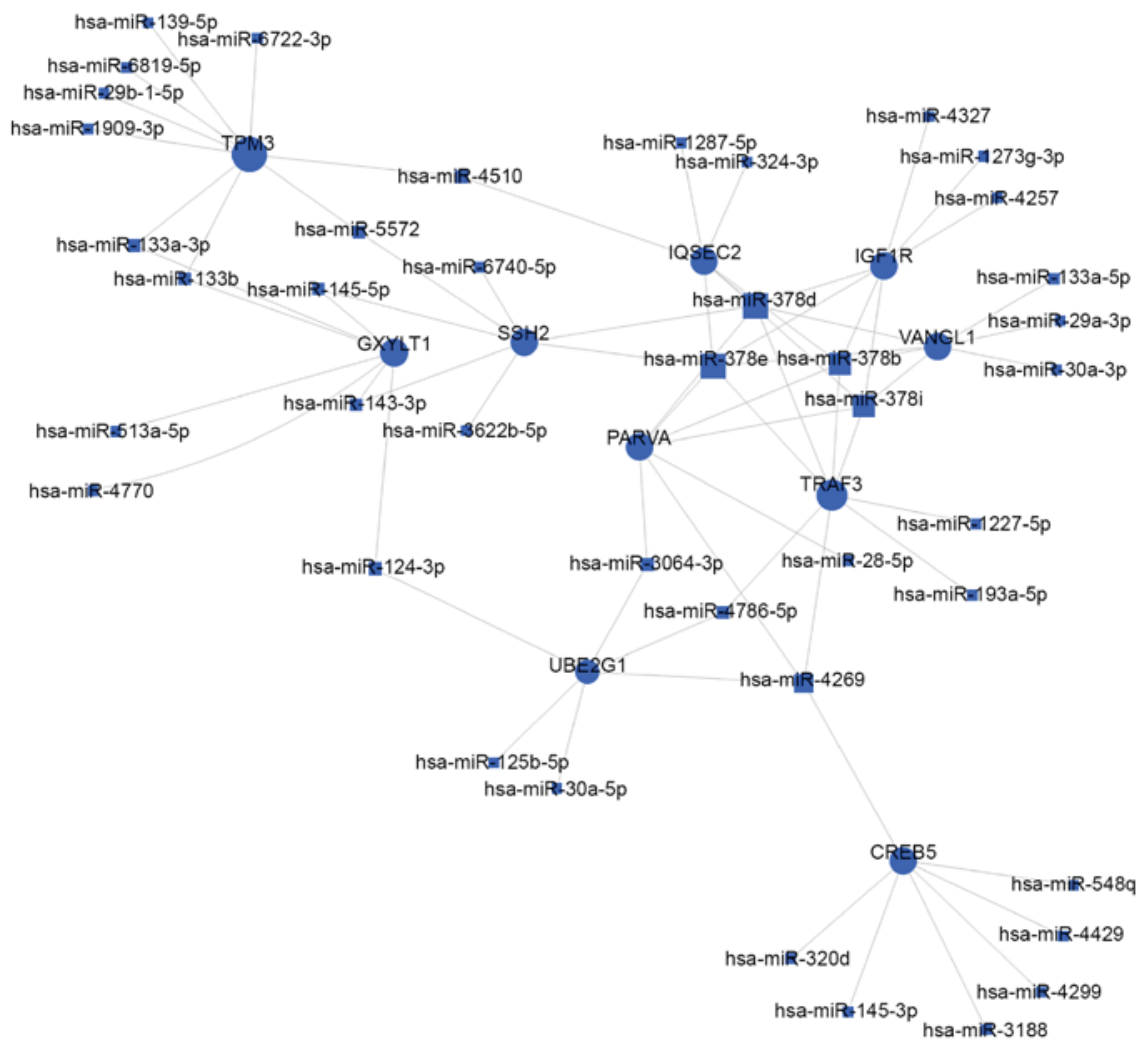

Figure 4. miRNAs-gene-network. According to the interactions between miRNAs and the intersected target genes, miRNAs-gene-network was constructed to illustrate the key regulatory functions of the identified miRNAs and their target genes. The blue circles represent genes, while blue square nodes represent downregulated miRNAs. The size of the circle or square node represents the degree value. A higher degree of gene/miRNAs indicates that it plays a more important role in the signaling network. miRNA/miR, microRNA.

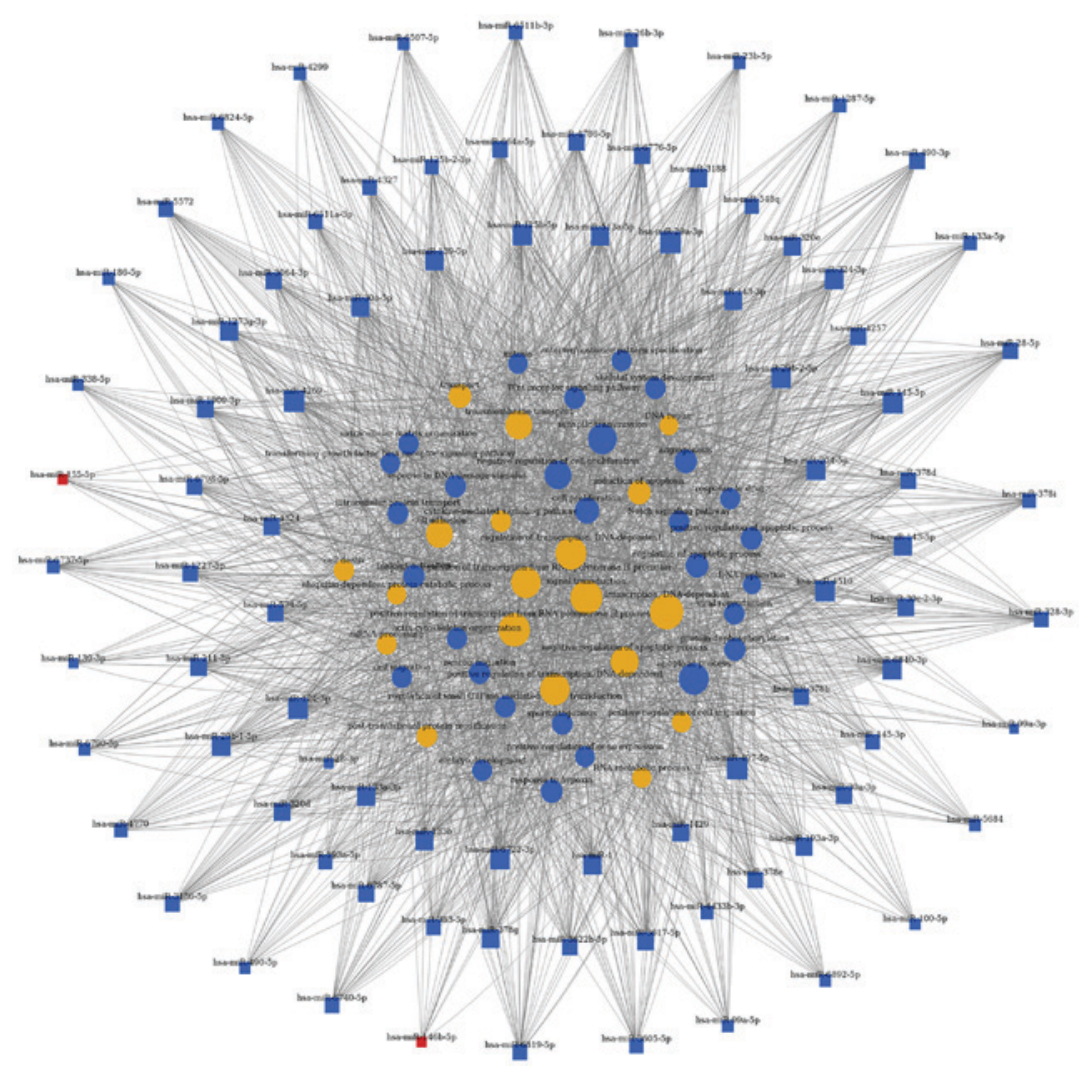

Figure 5. miRNAs-GO-network. The miRNAs-GO-network was generated according to the relationship of significant biological functions and miRNAs. The yellow and blue circles represent GOs, red square nodes represent upregulated miRNAs, and blue square nodes represent downregulated miRNAs. The size of the circle or square node represents the degree value. A higher degree of GO/miRNAs indicates that it plays a more important role in the signaling network. GO, gene ontology; miRNA/miR, microRNA. 
Table III. The top 10 miRNAs with high degrees of miRNAs-gene-networks and miRNAs-GO-networks.

\begin{tabular}{|c|c|c|c|c|c|c|}
\hline Rank & miRNAs & $\begin{array}{l}\text { miRNA-gene- } \\
\text { networks degree }\end{array}$ & Feature $^{\mathrm{b}}$ & miRNAs & $\begin{array}{c}\text { miRNA-GO- } \\
\text { networks degree }^{\mathrm{a}}\end{array}$ & Feature $^{\mathrm{b}}$ \\
\hline 1 & hsa-miR-497-5p & 123 & Down & hsa-miR-497-5p & 637 & Down \\
\hline 2 & hsa-miR-29a-3p & 88 & Down & hsa-miR-29a-3p & 518 & Down \\
\hline 3 & hsa-miR-124-3p & 84 & Down & hsa-miR-124-3p & 516 & Down \\
\hline 4 & hsa-miR-4269 & 64 & Down & hsa-miR-204-5p & 494 & Down \\
\hline 5 & hsa-miR-145-5p & 63 & Down & hsa-miR-4269 & 458 & Down \\
\hline 6 & hsa-miR-204-5p & 62 & Down & hsa-miR-145-5p & 430 & Down \\
\hline 7 & hsa-miR-4510 & 61 & Down & hsa-miR-6840-3p & 406 & Down \\
\hline 8 & hsa-miR-125b-5p & 57 & Down & hsa-miR-4510 & 404 & Down \\
\hline 9 & hsa-miR-6840-3p & 50 & Down & hsa-miR-6722-3p & 387 & Down \\
\hline 10 & hsa-miR-6722-3p & 47 & Down & hsa-miR-1 & 383 & Down \\
\hline
\end{tabular}

${ }^{\mathrm{a}}$ Changes of miRNA expression in MIBC compared with normal bladder. ${ }^{\mathrm{b}}$ Total number of connections around the miRNA. miRNA/miR, microRNA; GO, gene ontology; Down, downregulated in muscle invasive bladder cancer.

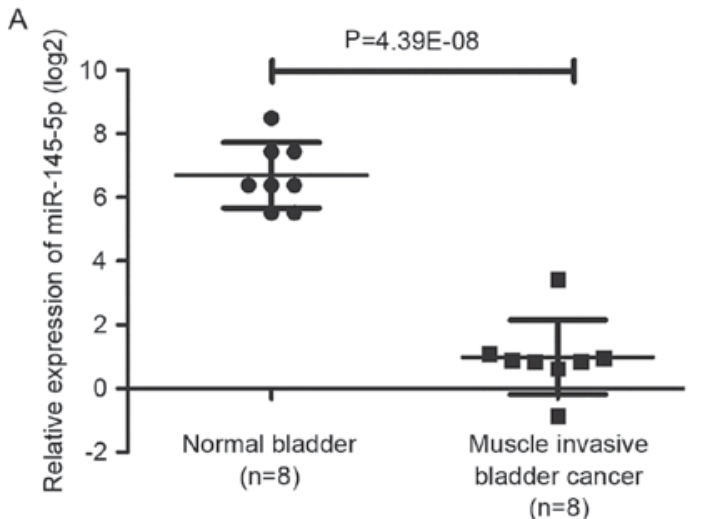

C

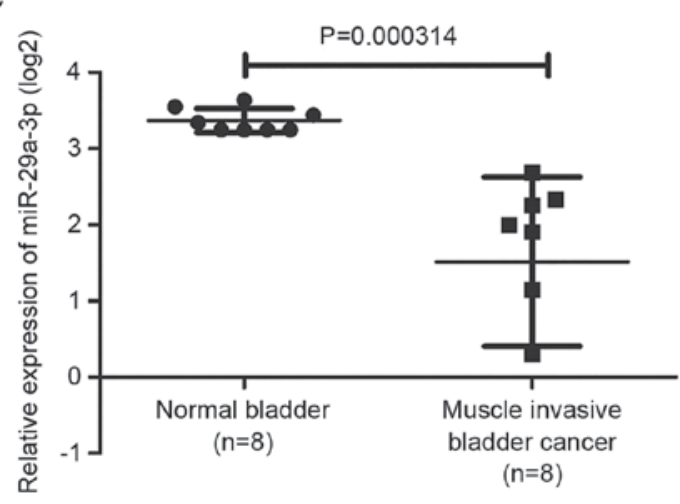

B
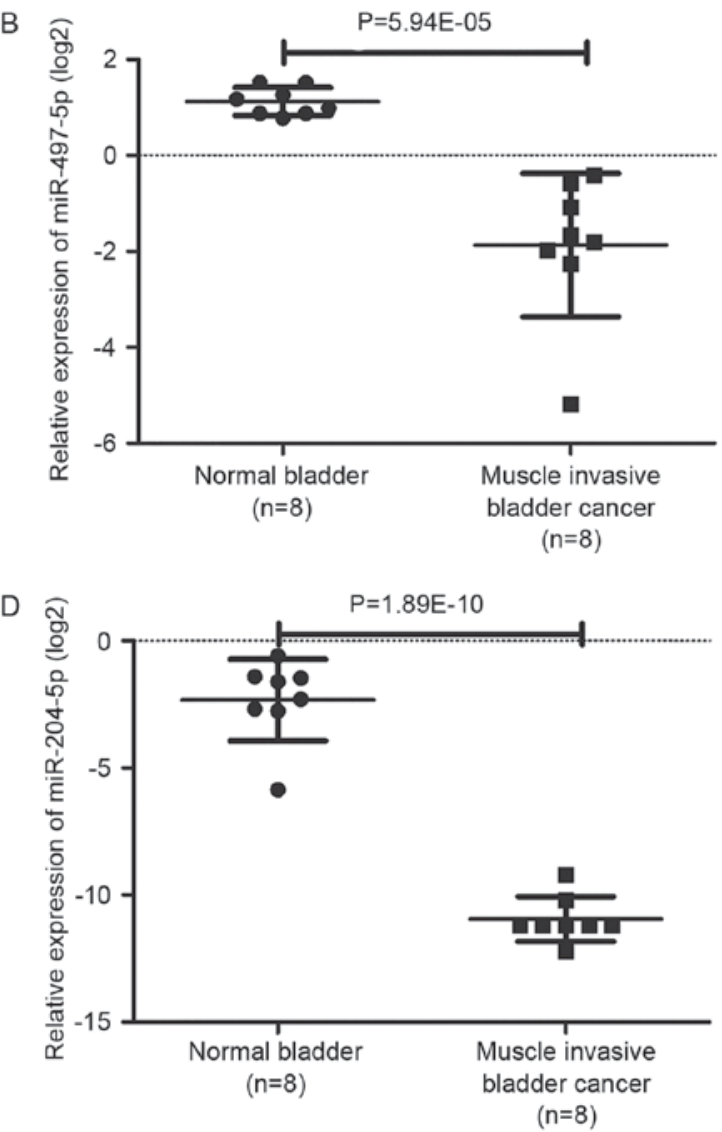

Figure 6. Validation of candidate miRNAs. Among the top rated nine miRNAs screened from our miRNA microarray, four miRNAs involving (A) hsa-miR-145-5p, (B) hsa-miR-497-5p, (C) hsa-miR-29a-3p and (D) hsa-miR-204-5p were also significantly altered in GSE40355. miRNA, microRNA.

hsa-miR-145-5p and hsa-miR-204-5p were also significantly altered in GSE40355.

\section{Discussion}

In the current study, by comparing array-based miRNA expression profiling performed on three MIBC tissues and three normal bladder tissues, the integrated bioinformatic analysis from miRNA expression profiling identified that 104 miRNAs were differentially expressed $(\mathrm{P}<0.05$, fold change $>1.5)$, of which, 102 were downregulated and 2 were upregulated (Fig. 1 and Table II). Among the top 10 fold change miRNAs, many of them were closely linked to occurrence and development of bladder cancer and might play vital role as oncogenes and 
Table IV. The alteration of top rated nine miRNAs in GSE76211 and GSE40355.

\begin{tabular}{|c|c|c|c|c|c|c|}
\hline \multirow[b]{2}{*}{ miRNAs } & \multicolumn{3}{|c|}{ GSE76211 } & \multicolumn{3}{|c|}{ GSE40355 } \\
\hline & Fold-change & P-value & Feature $^{a}$ & Fold-change & P-value & Feature $^{\mathrm{a}}$ \\
\hline hsa-miR-497-5p & -3.27 & 0.011865 & Down & -7.94 & $5.94 \times 10^{-5}$ & Down \\
\hline hsa-miR-29a-3p & -2.90 & 0.02804 & Down & -3.61 & 0.0003 & Down \\
\hline hsa-miR-124-3p & -1.70 & 0.025797 & Down & - & - & Down \\
\hline hsa-miR-4269 & -8.22 & 0.038312 & Down & - & - & Down \\
\hline hsa-miR-145-5p & -3.49 & 0.005372 & Down & -52.69 & $4.39 \times 10^{-8}$ & Down \\
\hline hsa-miR-204-5p & -6.00 & 0.015525 & Down & -463.33 & $1.89 \times 10^{-10}$ & Down \\
\hline hsa-miR-4510 & -2.80 & 0.004545 & Down & - & - & Down \\
\hline hsa-miR-6840-3p & -6.81 & 0.024616 & Down & - & - & Down \\
\hline hsa-miR-6722-3p & -2.90 & 0.011275 & Down & - & - & Down \\
\hline
\end{tabular}

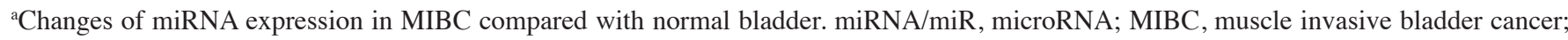
Down, downregulated in MIBC.

tumor suppressor genes, which has been published in several studies (23-27). Several studies have showed that miR-490-5p was significantly downregulated in BCa tissue samples compared with adjacent normal tissues (28). Low expression of miR-139-3p, miR-133, miR-145 and miR-1 in MIBC tissue samples compared with normal tissues was also reported by studies and played a functional role in bladder cancer cell lines (29-32).

Then, 11,884 genes were predicted as putative target genes of dysregulated miRNAs using target prediction method in GCBI online tools. According to the GO analysis, the predicted target genes were mainly involved in 'transcription, DNA-dependent', signal transduction, positive regulation of transcription from RNA polymerase II promoter, 'regulation of transcription, DNA-dependent' and axon guidance (Fig. 2A). Interestingly, the authors noticed two pairs of opposite GOs ('Negative regulation of transcription, DNA-dependent vs. Positive regulation of transcription, DNA-dependent' and 'Negative regulation of transcription from RNA polymerase II promoter vs. Positive regulation of transcription from RNA polymerase II promoter'). miRNAs were involved in post-transcriptional regulation of gene expression (33), which played a key role in various cellular processes including cellular differentiation, cell cycle progression and apoptosis. Among the predictive target genes of miRNAs, there may be lots of oncogenes and tumor suppressor genes. Oncogenes could promote BCa cell proliferation by positive regulation of transcription, in contrast, tumor suppressor genes could suppress BCa cell viability by negatively regulation of transcription. Therefore, it can be inferred that miRNA regulation disorders might account for these biological behaviors. As for biological pathways, the MAPK signaling pathway, apoptosis, pathways in cancer, cell cycle, p53 signaling pathway, calcium signaling pathway, Wnt signaling pathway, adherens junction, focal adhesion and Erbb signaling pathway were the top 10 enriched pathways of the predicted target genes (Fig. 2B). Many studies including our group (17-19) have ever reported that these pathways such as the MAPK signaling pathway, Wnt signaling pathway, p53 signaling pathway and Erbb signaling pathway play a functional role in human bladder cancer cells $(34,35)$. Besides, the Path-net analysis covering 36 significant pathways also showed that the MAPK signaling pathway, cell cycle, p53 signaling pathway, Wnt signaling pathway and calcium signaling pathway have a close correlation with bladder cancer, indicating that these pathways might play a key role in the development of human bladder cancer. In order to find out the key miRNAs, the authors conducted regulatory network analysis by overlapping significant miRNAs, pathways and GO analysis, revealing that the top nine miRNAs were hsa-miR-497-5p, hsa-miR-29a-3p, hsa-miR-124-3p, hsa-miR-4269, hsa-miR-145-5p, hsa-miR-204-5p, hsa-miR-4510, hsa-miR-6840-3p and hsa-miR-6722-3p. In order to validate that the nine candidate miRNAs identified were indeed dysregulated in BCa tissues, another miRNA microarray was performed by overlapping different expressed miRNAs and the top nine candidate miRNAs suggesting that four miRNAs involving hsa-miR-497-5p, hsa-miR-29a-3p, hsa-miR-145-5p and hsa-miR-204-5p were significantly altered. Moreover, as illustrated in Figs. 4 and 5, the top 10 target genes were TPM3, GXYLT1, SSH2, UBE2G1, CREB5, PARVA, TRAF3, IQSEC2, VANGL1 and IGF1R. Above all, these data showed that the regulatory network consisted of key miRNAs and genes may regulate biological process such as cell cycle, apoptosis and proliferation of human bladder cancer. Among the top ten target genes, tropomyosin 3 is a member of the tropomyosin family of actin-binding protein, which has been reported to relate to malignant transformation in BCa (36). VANGL planar cell polarity 1 (VANGL1), as an oncogene, is associated with many cancers. Park et al (37) revealed that miR-124 targeting VANGL could suppress colorectal cancer and $\mathrm{Oh}$ et al (38) reported that VANGL1 has correlation with tumor progression in human colorectal cancer. Insulin-like growth factor 1 receptor (IGF1R), with tyrosine kinase activity, binds insulin-like growth factor with a high affinity, playing a critical role in tumorigenesis and chemosensitivity $(39,40)$. In the present study, IGF1R has high correlation with has-miR-378 family. Some studies 
indicated that miR-378 deficiency played a key role in the development of cardiac hypertrophy by targeting IGF1R through negatively regulated Ras signaling pathway (41). Parvin alpha, a member of the parvin family of actin-binding proteins, playing a role in cell adhesion, motility and survival, is connected with different cancers, such as colorectal cancer and lung cancer (42).

The four miRNAs screened out from the miRNA microarray and verified by another miRNA microarray indeed had a functional role in MIBC, which has been demonstrated by previous studies. Zhang et al (43) reported that miR-497 was downregulated in $\mathrm{BCa}$ tissues compared with normal bladder tissues and may represent a novel prognostic biomarker for the early detection of metastasis of bladder cancer. Du et al (44) also reported that miR-497 was decreased in plasma of bladder cancer patients compared with healthy patients and could be a promising novel circulating biomarkers in clinical detection of bladder cancer. Chiyomaru et al (45) found that miR-145 was a tumor suppressor and inhibited cell viability by targeting FSCN1 in BCa cells. In addition, Avgeris et al (46) suggested that miR-145 could act as a novel marker helpful for prediction of oncologic outcome for bladder cancer patients. However, there are no related reports about miR-29a-3p and miR-204-5p in $\mathrm{BCa}$, therefore, the authors would like to investigate and confirm the two miRNAs using human BCa cell lines and mouse model in our next research article. However, predicting the miRNAs targets merely by bioinformatics analysis is not sufficient. Since the size of the MIBC samples used in the present study is small, these results may have many limitations. Thus, functional experiments should be performed strictly to verify the miRNAs and its targets in the further studies. As a result of that, the author group will select some of the significantly dysregulated miRNAs and perform verification experiments to confirm their targets and then figure out the functional role of miRNAs and the underlying mechanisms in MIBC.

\section{Acknowledgements}

The excellent technical assistance of Yuan Zhu, Shanshan Zhang and Danni Shan is gratefully acknowledged. The present study was supported in part by grants from the Zhongnan Hospital of Wuhan University Science, Technology and Innovation Seed Fund (grant no. cxpy20160031 and cxpy20160077) and the National Natural Science Foundation of China (grant no. 81300578). The funders had no role in study design, data collection and analysis, decision to publish, or preparation of the manuscript.

\section{References}

1. Griffiths TR; Action on Bladder Cancer: Current perspectives in bladder cancer management. Int J Clin Pract 67: 435-448, 2013.

2. Pastorelli R, Saletta F, Carpi D, Campagna R, dell'Osta C, Schiarea S, Vineis P, Airoldi L and Matullo G: Proteome characterization of a human urothelial cell line resistant to the bladder carcinogen 4-aminobiphenyl. Proteome Sci 5: 6, 2007.

3. Gu S, Jin L, Zhang F, Sarnow P and Kay MA: Biological basis for restriction of microRNA targets to the $3^{\prime}$ untranslated region in mammalian mRNAs. Nat Struct Mol Biol 16: 144-150, 2009.

4. Bartel DP and Chen CZ: Micromanagers of gene expression: The potentially widespread influence of metazoan microRNAs. Nat Rev Genet 5: 396-400, 2004
5. Hwang HW and Mendell JT: MicroRNAs in cell proliferation, cell death, and tumorigenesis. Br J Cancer 94: 776-780, 2006.

6. Nicoloso MS, Spizzo R, Shimizu M, Rossi S and Calin GA: MicroRNAs-the micro steering wheel of tumour metastases. Nat Rev Cancer 9: 293-302, 2009.

7. Adlakha YK and Saini N: Brain microRNAs and insights into biological functions and therapeutic potential of brain enriched miRNA-128. Mol Cancer 13: 33, 2014.

8. He Y, Lin J, Kong D, Huang M, Xu C, Kim TK, Etheridge A, Luo Y, Ding Y and Wang K: Current State of circulating MicroRNAs as cancer biomarkers. Clin Chem 61: 1138-1155, 2015.

9. Chawla JP, Iyer N, Soodan KS, Sharma A, Khurana SK and Priyadarshni P: Role of miRNA in cancer diagnosis, prognosis, therapy and regulation of its expression by Epstein-Barr virus and human papillomaviruses: With special reference to oral cancer. Oral Oncol 51: 731-737, 2015.

10. Andrew AS, Marsit CJ, Schned AR, Seigne JD, Kelsey KT, Moore JH, Perreard L, Karagas MR and Sempere LF: Expression of tumor suppressive microRNA-34a is associated with a reduced risk of bladder cancer recurrence. Int J Cancer 137: 1158-1166, 2015.

11. Bandres E, Agirre X, Ramirez N,Zarate R and Garcia-Foncillas J: MicroRNAs as cancer players: Potential clinical and biological effects. DNA Cell Biol 26: 273-282, 2007.

12. Catto JW, Miah S, Owen HC, Bryant H, Myers K, Dudziec E, Larré S, Milo M, Rehman I, Rosario DJ, et al: Distinct microRNA alterations characterize high- and low-grade bladder cancer. Cancer Res 69: 8472-8481, 2009.

13. Pignot G, Cizeron-Clairac G, Vacher S, Susini A, Tozlu S, Vieillefond A, Zerbib M, Lidereau R, Debre B, Amsellem-Ouazana D and Bieche I: microRNA expression profile in a large series of bladder tumors: Identification of a 3-miRNA signature associated with aggressiveness of muscle-invasive bladder cancer. Int J Cancer 132: 2479-2491, 2013.

14. Gottardo F, Liu CG, Ferracin M, Calin GA, Fassan M, Bassi P, Sevignani C, Byrne D, Negrini M, Pagano F, et al: Micro-RNA profiling in kidney and bladder cancers. Urol Oncol 25: 387-392, 2007.

15. Hidaka H, Seki N, Yoshino H, Yamasaki T, Yamada Y, Nohata N, Fuse M, Nakagawa $M$ and Enokida $H$ : Tumor suppressive microRNA-1285 regulates novel molecular targets: Aberrant expression and functional significance in renal cell carcinoma. Oncotarget 3: 44-57, 2012.

16. Yoshino H, Seki N, Itesako T, Chiyomaru T, Nakagawa M and Enokida $\mathrm{H}$ : Aberrant expression of microRNAs in bladder cancer. Nat Rev Urol 10: 396-404, 2013.

17. Cao R, Meng Z, Liu T, Wang G, Qian G, Cao T, Guan X, Dan H, Xiao Y and Wang X: Decreased TRPM7 inhibits activities and induces apoptosis of bladder cancer cells via ERK1/2 pathway. Oncotarget 7: 72941-72960, 2016.

18. Chen L, Wang G, Luo Y, Wang Y, Xie C, Jiang W, Xiao Y, Qian $\mathrm{G}$ and Wang X: Downregulation of LAPTM5 suppresses cell proliferation and viability inducing cell cycle arrest at G0/G1 phase of bladder cancer cells. Int J Oncol 50: 263-271, 2017.

19. Wang G, Cao R, Wang Y, Qian G, Dan HC, Jiang W, Ju L, Wu M, Xiao Y and Wang X: Simvastatin induces cell cycle arrest and inhibits proliferation of bladder cancer cells via PPARgamma signalling pathway. Sci Rep 6: 35783, 2016.

20. Tusher VG, Tibshirani R and Chu G: Significance analysis of microarrays applied to the ionizing radiation response. Proc Natl Acad Sci USA 98: 5116-5121, 2001.

21. Chu G, Seo M,Li j, Narasimhan B, Tibshirani R and Tusher V: SAM: Significance Analysis of Microarrays. Users Guide and technical document. http://statweb.stanford.edu/ tibs/SAM/sam.pdf.

22. Storey JD: A direct approach to false discovery rates. J Roy Stat Soc Ser 64: 479-498, 2002.

23. Li T, Pan H and Li R: The dual regulatory role of miR-204 in cancer. Tumour Biol 37: 11667-11677, 2016.

24. Yang G, Xiong G, Cao Z, Zheng S, You L, Zhang T and Zhao Y: miR-497 expression, function and clinical application in cancer. Oncotarget 7: 55900-55911, 2016.

25. Wang Y, Zhang X, Li H, Yu J and Ren X: The role of miRNA-29 family in cancer. Eur J Cell Biol 92: 123-128, 2013.

26. Wang X, Wu Q, Xu B, Wang P, Fan W, Cai Y, Gu X and Meng F: MiR-124 exerts tumor suppressive functions on the cell proliferation, motility and angiogenesis of bladder cancer by fine-tuning UHRF1. FEBS J 282: 4376-4388, 2015.

27. Shaham L, Binder V, Gefen N, Borkhardt A and Izraeli S: miR-125 in normal and malignant hematopoiesis. Leukemia 26: 2011-2018, 2012. 
28. Li S, Xu X, Xu X, Hu Z, Wu J, Zhu Y, Chen H, Mao Y, Lin Y, Luo J, et al: MicroRNA-490-5p inhibits proliferation of bladder cancer by targeting c-Fos. Biochem Biophys Res Commun 441: 976-981, 2013.

29. Yonemori M, Seki N, Yoshino H, Matsushita R, Miyamoto K, Nakagawa $\mathrm{M}$ and Enokida $\mathrm{H}$ : Dual tumor-suppressors miR-139-5p and miR-139-3p targeting matrix metalloprotease 11 in bladder cancer. Cancer Sci 107: 1233-1242, 2016.

30. Tao J, Wu D, Xu B, Qian W, Li P, Lu Q, Yin C and Zhang W: microRNA-133 inhibits cell proliferation, migration and invasion in prostate cancer cells by targeting the epidermal growth factor receptor. Oncol Rep 27: 1967-1975, 2012.

31. Kou B, Gao Y, Du C, Shi Q, Xu S, Wang CQ, Wang X, He D and Guo P: miR-145 inhibits invasion of bladder cancer cells by targeting PAK1. Urol Oncol 32: 846-854, 2014.

32. Yoshino H, Chiyomaru T, Enokida H, Kawakami K, Tatarano S, Nishiyama K, Nohata N, Seki $N$ and Nakagawa M: The tumour-suppressive function of miR-1 and miR-133a targeting TAGLN2 in bladder cancer. Br J Cancer 104: 808-818, 2011.

33. Ambros V: The functions of animal microRNAs. Nature 431: $350-355,2004$.

34. Pierzynski JA, Hildebrandt MA, Kamat AM, Lin J, Ye Y, Dinney CP and Wu X: Genetic Variants in the Wnt//-Catenin signaling pathway as indicators of bladder cancer risk. J Urol 194 1771-1776, 2015.

35. Lee SJ,Lim JH, Choi YH, Kim WJ and Moon SK: Interleukin-28A triggers wound healing migration of bladder cancer cells via NF- $x$ B-mediated MMP-9 expression inducing the MAPK pathway. Cell Signal 24: 1734-1742, 2012.

36. Pawlak G, McGarvey TW, Nguyen TB, Tomaszewski JE, Puthiyaveettil R, Malkowicz SB and Helfman DM: Alterations in tropomyosin isoform expression in human transitional cell carcinoma of the urinary bladder. Int J Cancer 110: 368-373, 2004.

37. Park SY, Kim H, Yoon S, Bae JA, Choi SY, Jung YD and Kim KK: KITENIN-targeting microRNA-124 suppresses colorectal cancer cell motility and tumorigenesis. Mol Ther 22 $1653-1664,2014$.
38. Oh HH, Park KJ, Kim N, Park SY, Park YL, Oak CY, Myung DS, Cho SB, Lee WS, Kim KK and Joo E: Impact of KITENIN on tumor angiogenesis and lymphangiogenesis in colorectal cancer. Oncol Rep 35: 253-260, 2016.

39. XM, Zhu HY, Bai WD, Wang T, Wang L, Chen Y, Yang AG and Jia LT: Epigenetic silencing of miR-375 induces trastuzumab resistance in HER2-positive breast cancer by targeting IGF1R. BMC Cancer 14: 134, 2014.

40. Taliaferro-Smith L, Oberlick E, Liu T, McGlothen T, Alcaide T, Tobin R, Donnelly S, Commander R, Kline E, Nagaraju GP, et al: FAK activation is required for IGF1R-mediated regulation of EMT, migration, and invasion in mesenchymal triple negative breast cancer cells. Oncotarget 6: 4757-4772, 2015.

41. Nagalingam RS, Sundaresan NR, Gupta MP, Geenen DL, Solaro RJ and Gupta M: A cardiac-enriched microRNA, miR-378, blocks cardiac hypertrophy by targeting Ras signaling. J Biol Chem 288: 11216-11232, 2013.

42. Huang AH, Pan SH, Chang WH, Hong QS, Chen JJ and Yu SL: PARVA promotes metastasis by modulating ILK signalling pathway in lung adenocarcinoma. PLoS One 10: e0118530, 2015.

43. Zhang Y, Zhang Z, Li Z, Gong D, Zhan B, Man X and Kong C: MicroRNA-497 inhibits the proliferation, migration and invasion of human bladder transitional cell carcinoma cells by targeting E2F3. Oncol Rep 36: 1293-1300, 2016.

44. Du M, Shi D, Yuan L, Li P, Chu H, Qin C, Yin C, Zhang Z and Wang M: Circulating miR-497 and miR-663b in plasma are potential novel biomarkers for bladder cancer. Sci Rep 5: 10437, 2015.

45. Chiyomaru T, Enokida H, Tatarano S, Kawahara K, Uchida Y, Nishiyama K, Fujimura L, Kikkawa N, Seki N and Nakagawa M: miR-145 and miR-133a function as tumour suppressors and directly regulate FSCN1 expression in bladder cancer. Br J Cancer 102: 883-891, 2010.

46. Avgeris M, Mavridis K, Tokas T, Stravodimos K, Fragoulis EG and Scorilas A: Uncovering the clinical utility of miR-143, miR-145 and miR-224 for predicting the survival of bladder cancer patients following treatment. Carcinogenesis 36: 528-537, 2015. 